\title{
Desingularizing homology manifolds
}

\author{
J BRYANT \\ S FERRY \\ W MIO \\ S WEINBERGER
}

\begin{abstract}
We prove that if $X^{n}, n \geq 6$, is a compact ANR homology $n$-manifold, we can blow up the singularities of $X$ to obtain an ANR homology $n$-manifold with the disjoint disks property. More precisely, we show that there is an ANR homology $n$-manifold $Y$ with the disjoint disks property and a cell-like map $f: Y \rightarrow X$.
\end{abstract}

$57 \mathrm{~N} 15,57 \mathrm{P} 99$

\section{Introduction}

Homology $n$-manifolds are defined as (finite-dimensional, locally contractible) spaces whose local homology $H_{*}(X, X-\{x\})$ is isomorphic to $H_{*}\left(\mathbb{R}^{n}, \mathbb{R}^{n}-\{0\}\right)$ for every $x \in X$. Equivalently, they can be described as spaces that satisfy a local form of Poincaré duality: every open set satisfies noncompact Poincaré duality between locally finite homology and cohomology. Originally introduced as a natural setting for the study of Poincaré duality, such spaces are important basic objects in geometric topology and arise in many investigations of structural properties of manifolds.

Until the 1990s, the only known non-manifold examples of homology manifolds were produced by singularizing manifolds: one would take disjoint, nearly contractible (technically, cell-like) subsets of a manifold and collapse them to points to obtain interesting singular spaces. Homology manifolds obtained in this manner are referred to as resolvable. A basic question then arises: Can every homology manifold be resolved by a manifold? In other words, given a homology $n$-manifold $X$, is there a topological $n$-manifold $M$ and a surjective map $f: M \rightarrow X$ that is cell-like? A map $f$ is cell-like if the inverse image of each point is contractible in every open neighborhood of itself. Quinn [19] showed that resolutions, if they exist (for $n>4$ ), are unique, and that existence is detected by an obstruction in $H^{0}(X ; Z)$ which is natural under restriction to open subsets. This implies that if a connected $X$ has even a single manifold point, then it is resolvable. 
A celebrated theorem of Edwards [9] asserts that a resolvable homology $n$-manifold, $n>5$, is a topological $n$-manifold if and only if it has a modicum of general position: continuous maps $f, g: D^{2} \rightarrow X$ of 2-disks into $X$ can be approximated by maps with disjoint images. This is known as the disjoint disks property, DDP. In other words, for resolvable homology manifolds, the DDP provides a geometric characterization of manifolds. In Bryant [3] it was shown that the DDP leads to general position properties in all dimensions.

In earlier work [4] we showed that nonresolvable homology manifolds exist and can be classified, up to s-cobordism, by a variant of surgery theory. We also conjectured that DDP homology manifolds share many geometric features with topological manifolds, notably, homogeneity. The first main goal of this paper is to show that all homology manifolds of dimension $\geq 6$ can be resolved by DDP homology manifolds. (In [4] we showed that they are all simple homotopy equivalent to DDP homology manifolds.) Even though the homogeneity conjecture remains open, one should view the "desingularization" in this paper as taming singularities by a resolution.

Alongside the DDP, which reflects a basic geometric characteristic of manifolds, another property that distinguishes manifolds among resolvable homology manifolds is the $U V^{1}$-approximation property. If one takes a degree- $k$ map $S^{n} \rightarrow S^{n}$, one naively expects a typical point inverse image to have at least $k$ components. The truth (see Bestvina [1]) is rather the opposite. If $n>4$, then $f$ is homotopic to a map with simply connected point inverses. This is predicted by the high connectivity of the homotopy fiber of the map. The point is that, rather than the usual strategy of approximating maps by smooth maps, it is sometimes more useful to consider approximations by maps that behave like space-filling "curves", which are closer models of the underlying abstract homotopy theory. The original construction of nonresolvable homology manifolds used this approach very strongly, and it is one of the themes of this paper.

Theorem A Every homology $n$-manifold $X, n \geq 6$, is the cell-like image of a DDP homology $n$-manifold that has the $U V^{1}$-approximation property.

(We shall not discuss here the sense in which the $U V^{1}$ map approximates a given map: smooth maps cannot be $C^{0}$-close to $U V^{1}$ maps in the case of degree $>1$ self maps of the sphere.)

An intriguing question presents itself of whether DDP is equivalent to the $U V^{1}$ approximation property in general. These are different types of conditions, but they both seem to pick out the nonsingular examples. In any case, the explicit focus on this property was critical to the constructions of resolutions of this paper, even if one was only interested in the DDP. 
Technically, this paper also proves a "squeezing" theorem in controlled geometric topology, which is new even in the case of manifolds.

Theorem B Suppose that $f: Y \rightarrow Z$ is a $U V^{1}$ map between compact, locally contractible, finite-dimensional spaces so that the inverse image of each open subset of $Z$ is a (usually noncompact) $n$-dimensional Poincaré space, $n \geq 6$. Then there is a total surgery obstruction $\sigma \in H_{n-1}(Z, Y ; \mathbb{L})$ that vanishes if and only if there exist a locally contractible, finite-dimensional DDP homology $n$-manifold $X$ and a map $g: X \rightarrow Y$ that is an $\epsilon$-homotopy equivalence over $Z$, for every $\epsilon>0$. Moreover, there is an $\epsilon_{0}>0$ depending on $Z$ so that $\sigma$ vanishes if there is an $\epsilon_{0}$-equivalence $g_{\epsilon_{0}}: X \rightarrow Y$ for some compact locally contractible, finite-dimensional homology $n$-manifold $X$.

Note that if $f: Y \rightarrow Z$ is the identity map, then this theorem reduces to our resolution theorem.

No doubt, the restriction to $U V^{1}$ maps can be substantially weakened. It is a very interesting question to wonder about how much. If the map $Y \rightarrow Z$ is a fibration, then the equivalence of the epsilon and the controlled statement (in the manifold case) is equivalent to the theorem of Chapman-Hughes [5; 14] that maps between manifolds that have an $\epsilon$-homotopy lifting property are near approximate fibrations. We expect that $\pi_{1}$ uniformity should suffice for the conclusion of our theorem, but we will not explore this aspect here.

Many of the ideas of this paper were already present in some form in our earlier paper, including the extensive use of controlled Poincaré duality, controlled surgery and the $U V^{1}$ approximation property of manifolds. However, the constructions here are both more efficient (we avoid Gromov-Hausdorff convergence of embeddings in Euclidean space and take a more conceptual approach to convergence problems) and more intricate (note the complexity of the statement of Theorem 8.1). The immediate technical problem to be confronted is that the sequence of approximate resolutions that were built in our previous paper in principle have different domains, and there is no immediate connection between them. A similar problem occurs in trying to produce controlled homotopy equivalences even for manifolds: the approximations are only close from the perspective of the control space, not from the perspective of the range. Essentially, we use some form of "squeezing", the $\alpha$-approximation theorem or the thin $h$-cobordism theorem to gain the extra control needed. Bryant and Mio were partially supported by NSF grants DMS-0071693 and DMS-9626624, Ferry was partially supported by NSF grants DMS-9971296 and DMS-9626101, and Weinberger was partially supported by NSF grant DMS-9803633. Ferry would also like to thank the University of Chicago for support during numerous visits. 


\section{Controlled maps}

Our first goal is to describe a class of metric spaces that we shall refer to as excellent spaces. These are ANR homology manifolds obtained by a variation of the construction presented in [4]. They are inverse limits of ever finer Poincare duality spaces, have the disjoint disks property and other useful properties. We begin by reviewing some basic facts about controlled maps.

\section{Definition 2.1}

(i) A compact metric space $X$ is $U V^{k}$ if whenever $X$ is embedded in a compact ANR, for each neighborhood $U$ of $X$ there is a smaller neighborhood $V$ of $X$ such that if $\alpha: S^{\ell} \rightarrow V, 0 \leq \ell \leq k$, there is an extension $\bar{\alpha}: D^{\ell+1} \rightarrow U$. Lacher [16] shows that this property can be checked on any given embedding of $X$ into an ANR.

(ii) Let $X$ be a metric space. A proper map $f: X \rightarrow Y$ is $U V^{k}$ if $f$ is surjective and $f^{-1}(y)$ is $U V^{k}$, for every $y \in Y$.

\section{Remarks}

(i) A proper map is $U V^{0}$ if and only if it is surjective and all of its point-inverses are connected. A proper $P L$ map is $U V^{k}$ if and only if it is surjective and its point-inverses are $k$-connected.

(ii) Compositions and uniform limits of $U V^{k}$ maps are $U V^{k}$.

We will need to use a characterization of $U V^{k}$ maps in terms of their lifting properties.

Definition 2.2 Let $X$ be an ANR and $p: Y \rightarrow B$ a map from a space $Y$ to a metric space $B$. Given $\epsilon>0$, a proper map $f: X \rightarrow Y$ is said to be $U V^{k}(\epsilon)$ over $B$ if whenever $(P, Q)$ is a polyhedral pair with $\operatorname{dim}(P) \leq k+1, \alpha_{0}: Q \rightarrow X$ is a map and $\alpha: P \rightarrow Y$ is a map with $f \circ \alpha_{0}=\alpha \mid Q$, there is a map $\bar{\alpha}: P \rightarrow X$ extending $\alpha_{0}$ so that $f \circ \bar{\alpha}$ is homotopic to $\alpha$ by a homotopy whose tracks have diameter $<\epsilon$ in $B$.

This is the same as Quinn's notion [18, Definition 5.1] of a relatively $(\epsilon, k+1)-$ connected map over $B$.

Lemma 2.3 Let $X$ and $Y$ be ANRs. A map $f: X \rightarrow Y$ is $U V^{k}$ if and only if $f$ is $U V^{k}(\epsilon)$ over $1_{Y}: Y \rightarrow Y$, for every $\epsilon>0$. The map $f$ is cell-like iff $f$ is $U V^{k}$ for all $k \leq \operatorname{dim}(X)$. 
Proof See Lacher [16].

Definition 2.4 Let $X$ and $Y$ be topological spaces and let $p: Y \rightarrow B$ a map to a metric space $B$. A map $f: X \rightarrow Y$ is an $\epsilon$-homotopy equivalence over $B$ if there exist a map $g: Y \rightarrow X$ and homotopies $h_{t}: X \rightarrow X, k_{t}: Y \rightarrow Y$ so that $h_{0}=1_{X}$, $h_{1}=g \circ f, k_{0}=1_{Y}, k_{1}=f \circ g, \operatorname{diam}\left\{p \circ k_{t}(y) \mid 0 \leq t \leq 1\right\}<\epsilon$ for every $y \in Y$, and $\operatorname{diam}\left\{p \circ f \circ h_{t}(x) \mid 0 \leq t \leq 1\right\}<\epsilon$ for every $x \in X$. The map $g$ is called an $\epsilon$-inverse for $f$.

By a 1-Lipschitz map between metric spaces we mean a Lipschitz map with Lipschitz constant 1 ie a map that does not increase distance.

\section{Lemma 2.5}

(i) If $\alpha: X \rightarrow Y$ in an $\epsilon$-homotopy equivalence over $Y$ with $\epsilon$-inverse $\beta$, and $\beta^{\prime}$ is $\delta$-homotopic to $\beta$ over $Y$, then $\beta^{\prime}$ is an $(\epsilon+\delta)$-inverse for $\alpha$.

(ii) If $\alpha_{i}: X_{i} \rightarrow X_{i-1}$ is a 1-Lipschitz $\epsilon_{i-1}$-equivalence, for $1 \leq i \leq n$, then $\alpha_{1} \circ \cdots \circ \alpha_{n}: X_{n} \rightarrow X_{0}$ is a $\left(3\left(\epsilon_{n-1}+\cdots+\epsilon_{1}\right)+\epsilon_{0}\right)$-equivalence.

The proof of the lemma is a simple exercise.

\section{Inverse systems}

Let $X_{1} \stackrel{\alpha_{2}}{\longleftarrow} X_{2} \stackrel{\alpha_{3}}{\longleftarrow} X_{3} \stackrel{\alpha_{4}}{\longleftarrow} \cdots$ be an inverse sequence of compact metric spaces and continuous maps, and let $X=\lim _{\longleftarrow} X_{i}$ be the inverse limit. We denote the projection $X \rightarrow X_{i}$ by $p_{i}$, and for $i>j$, we write $\alpha_{i, j}$ for the composition $\alpha_{j+1} \circ \cdots \circ \alpha_{i}: X_{i} \rightarrow$ $X_{j}$. For every $i, \alpha_{i, i}=\operatorname{id}_{X_{i}}$. We denote the metric on $X_{i}$ by $\rho_{i}$ and assume that $\operatorname{diam}\left(X_{i}\right) \leq 1$ for every $i$.

We equip $\bigsqcup X_{i}$, and hence the $X_{i}$ themselves, with a new system metric $d$ as follows

$$
d(x, y)= \begin{cases}\sum_{j=1}^{i} \frac{1}{2^{j}} \rho_{j}\left(\alpha_{i, j}(x), \alpha_{i, j}(y)\right) & \text { if } x, y \in X_{i} \\ d\left(\alpha_{i, j}(x), y\right)+\left(\frac{1}{2^{j}}-\frac{1}{2^{i}}\right) & \text { if } x \in X_{i}, y \in X_{j} \text { and } i>j .\end{cases}
$$

This metric on $\amalg X_{i}$ allows us to compare sizes in different $X_{i}$ s. In [4], we accomplished this by embedding all $X_{i}$ s under consideration in a euclidean space. Here, we achieve the same goal by introducing the metric $d$ that depends on the inverse system. 
Let $X_{i}^{+}$be the union of $X_{i}$ and a disjoint basepoint $*_{i}$ whose distance from each point in $X_{i}$ is 1 . For every $i$, the map

$$
x \rightarrow\left(\alpha_{i, 1}(x), \alpha_{i, 2}(x), \ldots, \alpha_{i, i-1}(x), x, *_{i+1}, *_{i+2}, \ldots\right),
$$

embeds $X_{i}$ into $\prod X_{i}^{+}$and the images of the $X_{i}$ are disjoint. These maps induce an isometric embedding of $\left(\bigsqcup X_{i}, d\right)$ into $\prod X_{i}^{+}$. The closure of $\bigsqcup X_{i}$ in $\prod X_{i}^{+}$is the union of the $X_{i}$ s with the inverse limit $X=\lim X_{i}$. Hence, the completion of $\bigsqcup X_{i}$

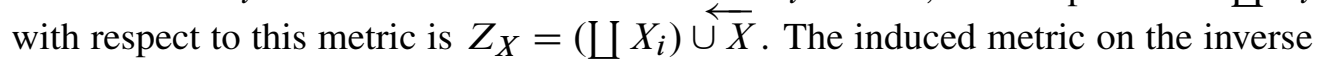
limit $X$ is given by

$$
d(x, y)=\sum_{i=1}^{\infty} \frac{1}{2^{i}} \rho_{i}\left(p_{i}(x), p_{i}(y)\right) .
$$

Notice that the projections $p_{i}: X \rightarrow X_{i}$ are 1-Lipschitz in the system metric.

If the bonding maps $\alpha_{i}$ are $\epsilon_{i-1}$-equivalences and $\sum \epsilon_{i}<\infty$, let $\beta_{i-1}: X_{i-1} \rightarrow X_{i}$ denote an $\epsilon_{i-1}$-inverse to $\alpha_{i}$. For $i \leq j$, define maps $\gamma_{i, j}: X_{i} \rightarrow X_{j}$ inductively by setting $\gamma_{i, i}=1_{X_{i}}$ and $\gamma_{i, j+1}=\beta_{j} \circ \gamma_{i, j}$. Then,

$$
\begin{aligned}
d\left(\gamma_{i, j}, \gamma_{i, j+1}\right) & =\frac{1}{2^{j+1}}+d\left(\gamma_{i, j}, \alpha_{j+1} \circ \beta_{j} \circ \gamma_{i, j}\right) \\
& =\frac{1}{2^{j+1}}+d\left(1_{X_{j}}, \alpha_{j+1} \circ \beta_{j}\right) \\
& \leq \frac{1}{2^{j+1}}+\epsilon_{j} .
\end{aligned}
$$

Thus, for each $i$, the sequence $\left\{\gamma_{i, j}\right\}_{j=i}^{\infty}$ is Cauchy and converges to a map $\gamma_{i}: X_{i} \rightarrow X$. Here, we are abusing notation: $X_{i}$ and $X$ are viewed as subspaces of $Z_{X}$. Combining these maps for $i \geq 1$, we obtain a retraction $\gamma: Z_{X}=\left(\amalg X_{i}\right) \cup X \rightarrow X$.

We form a space $T_{X}$ by inserting the mapping cylinder of $\alpha_{i}: X_{i} \rightarrow X_{i-1}$ between $X_{i}$ and $X_{i-1}$ in the space $Z_{X}$. Using the homotopy from $\beta_{i} \circ \alpha_{i+1}$ to the identity, we can extend $\gamma_{i, i+1}$ to a retraction from the mapping cylinder of $\alpha_{i+1}$ onto $X_{i+1}$. Combining these, we can extend $\gamma$ to a retraction of $T_{X}$ onto $X$.

Lemma 3.1 If the spaces $X_{i}$ are ANRs and the maps $\alpha_{i}$ are $\epsilon_{i-1}$-homotopy equivalences in the system metric with $\sum \epsilon_{i}<\infty$, then $X$ is an ANR. Moreover, $X$ is $\left(3 \sum_{j=i+1}^{\infty} \epsilon_{j}+\epsilon_{i}\right)$-equivalent to $X_{i}$.

Proof By Chapman-Siebenmann [6], $T_{X}$ is an ANR. Since $T_{X}$ retracts to $X, X$ is also an ANR. The second assertion follows from Lemma 2.5 (ii). Given $\delta>0$, $X$ is $\delta$-equivalent to $X_{k}$ for large $k$. The maps are given by pushing down the 
mapping cylinder and by retracting back into X. Choosing $k$ large, this implies that $X$ is $\left(3 \sum_{j=i+1}^{k} \epsilon_{j}+\epsilon_{i}+3 \delta\right)$-equivalent to $X_{i}$ for every $\delta>0$. The result follows.

Remark A similar argument shows that if each bonding map $\alpha_{i}$ is a $U V^{k}$-map, then each projection map $X \rightarrow X_{i}$ is a $U V^{k}$-map.

We will find it useful to construct new inverse systems by passing to subsequences. Let $\left(X_{i}, \alpha_{i}\right)_{i=1}^{\infty}$ be an inverse system and let $\left\{i_{j}\right\}_{j=1}^{\infty}$ be a strictly increasing sequence of positive integers. For each $j$, let $\rho_{i_{j}}^{\prime}$ be the metric on $X_{i_{j}}$ induced by the system metric $d$ on $Z_{X}$. The sequence $\left(X_{i_{j}}, \alpha_{i_{j}, i_{j-1}}\right)_{j=1}^{\infty}$ forms an inverse system of metric spaces and continuous maps with respect to the metrics $\rho_{i_{j}}^{\prime}$. The system metric $d^{\prime}$ for the subsystem is defined using the metric $\rho_{i_{j}}^{\prime}$ on $X_{i_{j}}$, for all $j$.

Lemma 3.2 If the maps $\alpha_{i}$ are $\epsilon_{i-1}$-equivalences in the system metric $d$, then the bonding maps $\alpha_{i_{j+1}, i_{j}}$ of the subsystem are $\left(3 \sum_{k=i_{j}+1}^{i_{j+1}-1} \epsilon_{k}+\epsilon_{i_{j}}\right)$-equivalences with respect to the metric $d^{\prime}$.

Proof Notice that $d^{\prime}(x, y) \leq d(x, y)$, for any $x, y \in \bigsqcup X_{i_{j}}$, since the bonding maps of any inverse system are 1-Lipschitz in the system metric. Hence, the result follows from Lemma 2.5 (ii).

\section{Controlled Poincaré duality}

Let $f: E \rightarrow B$ be a continuous map from a topological space to a compact connected metric space. Let $\mathcal{E}(f)=\left\{(e, \omega) \in E \times B^{I} \mid f(e)=\omega(0)\right\}$. Given $b \in B$, let $\omega_{b}$ denote the constant path at $b$. We can identify $E$ with a subspace of $\mathcal{E}(f)$ via the map $e \mapsto\left\{\left(e, \omega_{f(e)}\right)\right\}$. There is a projection $\mathcal{P}_{f}: \mathcal{E}(f) \rightarrow B$ given by $(e, \omega) \rightarrow \omega(1)$ and the diagram

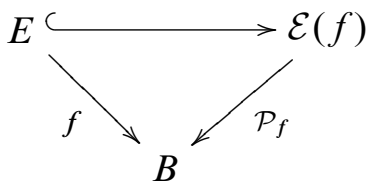

is commutative. $\mathcal{P}_{f}$ is a Hurewicz fibration known as the path fibration associated to $f$. The fiber of $\mathcal{P}_{f}$ is called the homotopy fiber of $f$.

Definition 4.1 $f: E \rightarrow B$ is an $\epsilon$-fibration if there is a retraction $r: \mathcal{E}(f) \rightarrow E$ such that $d\left(f \circ r, \mathcal{P}_{f}\right)<\epsilon$. The map $f: E \rightarrow B$ is an approximate fibration if it is an $\epsilon$-fibration for every $\epsilon>0$. More generally, if $q: B \rightarrow Z$ is a map to a metric space $Z$, we say that $f: E \rightarrow B$ is an $\epsilon$-fibration over $Z$ if $d\left(q \circ f \circ r, q \circ \mathcal{P}_{f}\right)<\epsilon$. 
Lemma 4.2 $A$ map $f: E \rightarrow B$ is an $\epsilon$-fibration over the metric space $Z$ if and only if for any commutative diagram

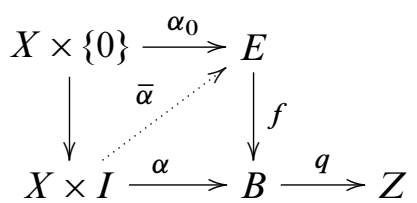

there is a map $\bar{\alpha}: X \times I \rightarrow E$ such that $\left.\bar{\alpha}\right|_{X \times\{0\}}=\alpha_{0}$ and $d(q \circ \alpha, q \circ f \circ \bar{\alpha})<\epsilon$.

Proof $(\Rightarrow)$ There is a lift $\alpha^{\prime}: X \times I \rightarrow \mathcal{E}(f)$ such that $\mathcal{P}_{f} \circ \alpha^{\prime}=\alpha$ and $\left.\alpha^{\prime}\right|_{X \times\{0\}}=\alpha_{0}$. Let $\bar{\alpha}=r \circ \alpha^{\prime}$. Then,

$$
d(q \circ f \circ \bar{\alpha}, q \circ \alpha)=d\left(q \circ f \circ r \circ \alpha^{\prime}, q \circ \mathcal{P}_{f} \circ \alpha^{\prime}\right) \leq d\left(q \circ f \circ r, q \circ \mathcal{P}_{f}\right)<\epsilon .
$$

$(\Leftarrow)$ Consider the diagram

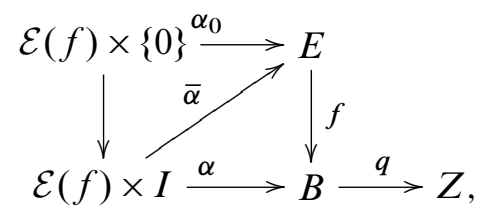

where $\alpha(e, \omega, t)=\omega(t), \alpha_{0}(e, \omega)=\left(e, \omega_{f(e)}\right)$ and $\bar{\alpha}$ is an $\epsilon$-lift of $\alpha$ over $Z$. Let $r(e, \omega)=\bar{\alpha}(e, \omega, 1)$. The map $r$ is a retraction because we can arrange as in Dugundji [8] that constant paths lift to constant paths. Since $\alpha(e, \omega, 1)=\mathcal{P}_{f}(e, \omega)$, it follows that $d\left(q \circ f \circ r, q \circ \mathcal{P}_{f}\right)<\epsilon$.

Let $X$ be a compact ANR. A pair $(N, X)$ is an abstract mapping cylinder neighborhood of $X$ if $N$ is a compact topological manifold containing $X$ in its interior, and there is a $U V^{1}$ map $p: \partial N \rightarrow X$ such that $(N, X)$ is homeomorphic to $\left(C_{p}, X\right)$, where $C_{p}$ is the mapping cylinder of $p$.

Definition 4.3 A finite-dimensional ANR $X$ is an $\epsilon$-Poincaré duality space if $X$ has an abstract mapping cylinder neighborhood $(N, X)$ such that $p: \partial N \rightarrow X$ is an $\epsilon$-fibration with homotopy fiber homotopy equivalent to $S^{k}$, for some $k \geq 2$. If $p: \partial N \rightarrow X$ is an approximate fibration, then $X$ is said to be a controlled Poincaré duality space over itself.

Remark The definition given here differs from the one given in [4] but is equivalent to it. That approximate spherical fibration (the definition we use here) follows from the chain duality (assumed in [4]) is proven on page 446-448 of that paper. On the other hand, the approximate chain level Poincaré duality definition follows directly from the 
current definition by combining Thom isomorphism with Poincaré duality. The Thom isomorphism theorem for the spherical fibration that we assume identifies the cochain complex of the space with that of its mapping cylinder neighborhood (up to a shift by codimension), and the latter is identified, by Poincare duality, with the chain complex of the neighborhood, which is homotopy equivalent to that of the original space.

Proposition 4.4 (Daverman-Husch [7]) Let $X$ be a finite-dimensional compact ANR. If $X$ is a controlled Poincaré duality space over itself, then $X$ is a homology manifold.

The converse statement is also valid, but will not be needed in this paper.

The next ingredient is a slight rewording of [4, Propostion 4.6]. The only difference between the two versions is that this version is an " $\epsilon-\delta$ " statement while the previous version is a " $T \epsilon-\epsilon$ " statement for some constant $T$. This change is needed because the control space in the present version is a compact ENR rather than a finite polyhedron.

Proposition 4.5 (Bryant et al [4]) Let $X$ be a compact ENR and let $n$ be given. For every $\epsilon>0$ there is a $\delta>0$ so that if

(i) $\left(M_{1}, \partial M_{1}\right)$ and $\left(M_{2}, \partial M_{2}\right)$ are orientable $n$-manifolds.

(ii) $p_{1}: M_{1} \rightarrow X$ and $p_{2}: M_{2} \rightarrow X$ are $U V^{1}$-maps with $p_{i} \mid \partial M_{i}$ a $U V^{0}-$ map for $i=1,2$.

(iii) $h$ : $\partial M_{1} \rightarrow \partial M_{2}$ is an orientation-preserving $\delta$-equivalence over $X$ with $p_{2} \circ h \delta-$ close to $p_{1}$.

then there is a $U V^{1}$-map $p: M_{1} \cup_{h} M_{2} \rightarrow X$ that is an $\epsilon$-Poincaré duality space over $X$. Moreover, $p$ is $\epsilon$-close to $p_{i}$ for $i=1,2$.

\section{Excellent metric spaces}

Definition 5.1 Let $B$ be a compact metric space. A pleasant $\epsilon$-controlled 2-patch space over $B$ is a pair $(X, p)$, where $p: X \rightarrow B$ is a $U V^{1}$ map and $X=S \cup_{f} T$ is a space obtained by gluing together two compact $n$-manifolds $S$ and $T$ (the patches of $X$ ) by a $U V^{1} \epsilon$-homotopy equivalence $f: \partial S \rightarrow \partial T$ (over $B$ ) of their boundaries. We also require that the maps $p|T, p| \partial T$ and the composition $S \rightarrow X=S \cup_{f} T \rightarrow B$ be $U V^{1}$. Finally, we require that every map of a sphere $S^{k}$ into $X$ be $\epsilon$-homotopic to a map $S^{k} \rightarrow T$, when $k \leq[n / 2]$. 
Definition 5.2 A compact metric space $X$ is excellent if there exist a sequence $\left\{\epsilon_{i}\right\}$ with $\epsilon_{i}>0$ and $\sum \epsilon_{i}<\infty$, and an inverse system $\left(X_{i}, \alpha_{i}\right)$ such that

(i) $X=\lim _{S_{i}}\left(X_{i}, \alpha_{i}\right)$, where each $\left(X_{i}, \alpha_{i}\right)$ is a pleasant $\epsilon_{i}$-controlled 2-patch space $X_{i}=\overleftarrow{S_{i}} \cup_{f_{i}} T_{i}$ over $X_{i-1}$

(ii) Each $\alpha_{i}$ is a $U V^{1} \epsilon_{i-1}$-equivalence.

Here, all sizes are measured using the system metric $d$.

Lemma 5.3 If $M^{n}$ and $N^{n}$ are compact manifolds with boundary, $n \geq 5$, and $f: \partial M \rightarrow \partial N$ is a $U V^{1}$ map, then $X=M \cup_{f} N$ has the disjoint disks property.

Proof Let $\alpha_{1}, \alpha_{2}: D^{2} \rightarrow X$ be continuous maps. The singular set of $X$ has a neighborhood homeomorphic to $\partial M \times[-1,0] \cup_{f} \partial N \times[0,1]$. By making the image of the disks transverse to $\partial M \times\{-\epsilon\}$ and pushing along the product structure in the positive direction, one may arrange that the intersection $K_{i}$ of $\alpha_{i}\left(D^{2}\right)$ and the singular set is 1-dimensional, $i=1,2$. Pushing $K_{1}$ and $K_{2}$ apart in $\partial N \times\{0\}$, use the lifting property of $U V^{1}$ maps and the estimated homotopy extension theorem [4] to extend this homotopy to obtain small deformations $\bar{\alpha}_{1}$ and $\bar{\alpha}_{2}$ of $\alpha_{1}$ and $\alpha_{2}$, respectively, with the property that $\bar{\alpha}_{1}\left(D^{2}\right) \cap \bar{\alpha}_{2}\left(N_{2}\right)=\varnothing$ and $\bar{\alpha}_{2}\left(D^{2}\right) \cap \bar{\alpha}_{1}\left(N_{1}\right)=\varnothing$, where $N_{i}$ is a small regular neighborhood of $K_{i}$ in $D^{2}, i=1,2$. Now, using the DDP on the complement of the singular set of $X$ gives us a small separation of the disks in $X$.

Proposition 5.4 If $X$ is an excellent metric space of dimension $n \geq 5$, then $X$ is an ANR homology $n$-manifold with the disjoint disks property.

Proof Let $\left\{X_{i}, \alpha_{i}\right\}$ be an inverse system of 2-patch spaces exhibiting $X$ as an excellent metric space, where $\alpha_{i+1}: X_{i+1} \rightarrow X_{i}$ an $\epsilon_{i}$-equivalence. By Lemma 3.1, $X=\lim X_{i}$ is an ANR. It is easy to check that the inverse limit of ANRs with the DDP and $\overleftarrow{U} V^{1}$ maps has the DDP. Hence, $X$ has the DDP since, by Lemma 5.3, each $X_{i}$ has the DDP. To complete the proof of the proposition, we need to show that $X$ is a homology manifold. The argument will be similar to the one presented in [4, Section 5].

Embed $T_{X}$ tamely in a high-dimensional euclidean space and let $W_{1}$ be a mapping cylinder neighborhood. For each $i$, let $T_{X_{i}}$ be the part of $T_{X}$ between $X_{i}$ and $X$, and let $W_{i}$ be a small mapping cylinder neighborhood of $T_{X_{i}}$ with $W_{i} \subset W_{i-1}$. For any $i>1$, the region between $W_{1}$ and $W_{i}$ has a product structure. Let $\left\{\delta_{i}, i \geq 1\right\}$ be a sequence of positive real numbers with $\sum \delta_{i}<\infty$. If $i_{2}<i_{3}$ are large enough positive integers, by the thin $h$-cobordism theorem (see Quinn [18]), the region between $W_{i_{2}}$ and $W_{i_{3}}$ has a $\delta_{1}$-controlled product structure over $X_{1}$ since $\alpha_{i_{3}, i_{2}}: X_{i_{3}} \rightarrow X_{i_{2}}$ is a fine 
equivalence over $X_{1}$. Arguing inductively and passing to an appropriate subsequence, one can assume that the region $V_{i}$ between $W_{i}$ and $W_{i+1}$ has a $\delta_{i}$-controlled product structure over $X_{i-1}$, for $i>1$. Deforming $W_{1}-\operatorname{int}\left(W_{i}\right)$ to $\partial W_{i}$ along the product structures on $V_{1}, \ldots, V_{i-1}$ and composing with the regular neighborhood projection $W_{i} \rightarrow X_{i}$, we obtain a spherical fine approximate fibration structure on $\rho_{i}^{\prime}: \partial W_{1} \rightarrow X_{i}$ that induces a mapping cylinder structure on the neighborhood $W_{1}$ of $X_{i}$.

Let $\rho_{i}: \partial W_{1} \rightarrow X$ denote the composition

$$
\partial W_{1} \stackrel{\rho_{i}^{\prime}}{\rightarrow} X_{i} \stackrel{\gamma_{i}}{\rightarrow} X
$$

Since $\sum \delta_{i}<\infty$, the sequence $\rho_{i}$ converges to a map $\rho: \partial W_{1} \rightarrow X$ that induces a controlled Poincaré duality structure on $X$, since, by [4, Proposition 4.6], each $X_{i}$ is a fine Poincare space over $X_{i-1}$. Hence, by Proposition $4.4, X$ is a homology manifold.

\section{Constructing $U V^{k}$ maps}

A deformation theorem of Bestvina and Walsh states that, below middle and adjacent dimensions, $(k+1)$-connected mappings of manifolds to polyhedra can be deformed to $U V^{k}$ mappings. The proof of our main result uses a controlled analogue of this result. We will first present an alternative proof of the Bestvina-Walsh theorem and then show how the arguments can be modified to yield a controlled version. Kawamura [15] remarks that this controlled theorem can also be proven using the techniques of Bestvina and Walsh.

Definition 6.1 A map $f: X \rightarrow Y$ is $k$-connected if $f_{*}: \pi_{\ell}(X) \rightarrow \pi_{\ell}(Y)$ is an isomorphism for $\ell<k$ and an epimorphism for $\ell=k$. This is the same as saying that $\pi_{\ell}(f)=\pi_{\ell}\left(C_{f}, X\right)=\{0\}$, for $0 \leq \ell \leq k$, where $C_{f}$ is the mapping cylinder of $f$.

Theorem 6.2 (Bestvina and Walsh [1]) Let $M^{m}$ be a compact manifold and $K$ a polyhedron. If $f: M \rightarrow K$ is a $(k+1)$-connected map and $f \mid \partial M$ is $U V^{k}$, then $f$ is homotopic rel $(\partial M)$ to a $U V^{k}$ map, provided that $k \leq\left[\frac{m-3}{2}\right]$.

Other results of this type are due to Keldyš, Anderson, Wilson, Walsh, Černavskii and Ferry. We begin the proof by recalling a theorem of Černavskii.

Theorem 6.3 (Černavskii [21]) If $M^{m}$ is a manifold and $k \leq\left[\frac{m-3}{2}\right]$, then there is a $U V^{k}$ map $p: M \rightarrow M \times I$. Moreover, for any $\epsilon>0$, we can choose $p$ so that proj $\circ p$ is $\epsilon$-close to $1_{M}: M \rightarrow M$. 

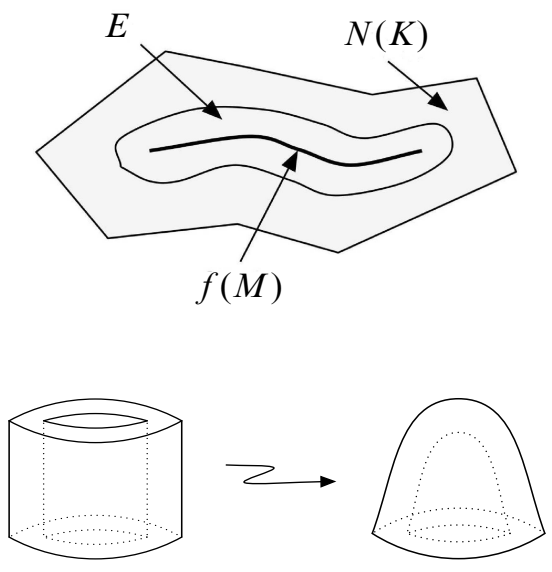

Iterating Černavskii's construction, we obtain $U V^{k}$ maps of $M$ onto $M \times I^{\ell}$, for any $\ell$, and also onto any disk bundle $E$ over $M$. This last follows from writing $E$ as a direct summand of a trivial bundle, mapping $M$ onto the trivial bundle and then projecting back to $E$.

Proof of the absolute case of the Bestvina-Walsh theorem Let $f: M \rightarrow K$ be as in the statement of the theorem and let $N(K)$ be a regular neighborhood of $K$ in some euclidean space $\mathbb{R}^{n}, n \geq 2 m+3$. Compose $f$ with the inclusion $K \hookrightarrow N(K)$ to obtain a map $g: M \rightarrow N(K)$. After a homotopy, we may assume that $g$ is an embedding and that $g(M)$ has a normal disk bundle $E$ in $N(K)$ (see Hirsch [13]). Using Černavskii's process, construct a $U V^{k}$ map $\phi: M \rightarrow E$. Since compositions of $U V^{k}$ maps are $U V^{k}$, we will be done if we can construct $U V^{k}$ map $\psi: E \rightarrow N(K)$, because composing $\psi \circ \phi$ with the cell-like regular neighborhood collapse $N(K) \rightarrow K$ will give a $U V^{k}$ map $M \rightarrow K$ homotopic to $f$.

By Lemma 6.4 below, the inclusion $\partial E \rightarrow N(K)-\stackrel{\circ}{E}$ is $(k+1)-$ connected. The usual handle-trading lemma (see Rourke-Sanderson [20, Lemmas 6.14-6.16]) implies that we can find a handle decomposition

$$
N(K)-\stackrel{\circ}{E} \cong \partial E \times I \cup\{(k+2)-\text { handles }\} \cup\{(k+3)-\text { handles }\} \cup \ldots
$$

To complete the proof of the theorem, we use the fact that there is a simple $U V^{r}$ map from a manifold $V^{n+1}$ to the same manifold with an $(r+2)$-handle attached to the boundary. Write $V$ as $V \cup\left(S^{r+1} \times D^{n-r-1} \times I\right)$ and collapse $S^{r+1} \times D^{n-r-1} \times I$ to $V \cup(r+2)$-handle by a map whose only non-trivial point-inverses are spheres of dimension $r+1$. 
Composing such maps for the handles of the decomposition of $N(K)-\stackrel{\circ}{E}$ given above, we obtain a $U V^{k}$ map $E \rightarrow N(K)$.

Lemma 6.4 The inclusion $\partial E \rightarrow N(K)-\stackrel{\circ}{E}$ is $(k+1)$-connected.

Proof Let $\alpha:\left(D^{s}, S^{s-1}\right) \rightarrow(N(K)-\stackrel{\circ}{E}, \partial E)$ be a map, $0 \leq s \leq k+1$. Including in $(N(K), E)$ and using the $(k+1)$-connectivity, we obtain a homotopy $\bar{\alpha}_{t}:\left(D^{s}, S^{s-1}\right) \rightarrow(N(K), E)$ so that $\bar{\alpha}_{0}=\alpha, \bar{\alpha}_{1}\left(D^{s}, S^{s-1}\right) \subseteq E$ and $\bar{\alpha}_{t} \mid S^{s-1}=$ $\alpha \mid S^{s-1}$ for all $t$. By general position, we may assume that $\bar{\alpha}_{t}\left(D^{s}\right) \cap f(M)=\varnothing$ for all $t$. Composing $\bar{\alpha}$ with the retraction $r: N(K)-f(M) \rightarrow N(K)-\stackrel{\circ}{E}$ gives a homotopy which shows that $[\alpha]$ is trivial in $\pi_{S}(N(K)-\stackrel{\circ}{E}, \partial E)$.

We now state our controlled analogue of the Bestvina-Walsh theorem.

Theorem 6.5 For any non-negative integers $m, k$ and $d$ satisfying $k \leq\left[\frac{m-3}{2}\right]$, there is a constant $C(m, k, d)>0$ such that if $M^{m}$ is a compact connected manifold and $K$ is a connected $d$-dimensional polyhedron, then every map $f: M \rightarrow K$ that is $U V^{k}(\delta)$ over some metric space $B$ is $C(m, k, d) \cdot \delta$-homotopic over $B$ to a $U V^{k}$ map.

Proof Suppose that $f: M \rightarrow K$ is a $U V^{k}(\delta)$ map over $B$. Let $N^{\prime}(K)$ be a regular neighborhood of $K$ in $\mathbb{R}^{2 d+1}$. Thicken $N^{\prime}(K)$ by taking a product with $I^{2 m+1}$ to obtain a regular neighborhood $N(K)$ of $K$ in $\mathbb{R}^{2 m+2 d+2}$ and a projection $c: N(K) \rightarrow$ $K$. We abuse notation and refer to the composition

$$
M \stackrel{f}{\rightarrow} K \hookrightarrow N(K)
$$

as $f$. We can perturb $f$ along the $I^{2 m+1}$-coordinate to an embedding $f^{\prime}$. This ensures that $c \circ f^{\prime}=f$. Let $E$ be a disk bundle neighborhood of $f^{\prime}(M)$ in $N(K)$.

Claim If $E$ is chosen to be a thin enough neighborhood of $f^{\prime}(M)$, then $(N(K)-$ $\stackrel{\circ}{E}, \partial E)$ is $(\delta, k+1)$-connected over $B$ in the following sense: if $\alpha:\left(D^{s+1}, S^{s}\right) \rightarrow$ $(N(K)-\stackrel{\circ}{E}, \partial E)$ is a map and $0 \leq s \leq k$, then there is a homotopy $\alpha_{t}$ starting with $\alpha$ and ending with a map $\alpha_{1}:\left(D^{s+1}, S^{s}\right) \rightarrow(\partial E, \partial E) \subset(N(K)-E, \partial E)$ such that the tracks of the homotopy have diameter $<\delta$ when projected to $B$.

To prove the claim, first note that $f: M \rightarrow N(K)$ is $U V^{k}(\delta)$ over $B$. Given a polyhedral pair $(P, Q)$ and maps $\beta_{0}: Q \rightarrow M$ and $\beta: P \rightarrow N(K)$ with the property that $f^{\prime} \circ \beta_{0}=\beta$, consider the composition $c \circ \beta$. By the $U V^{k}(\delta)$ property, there is a map $\bar{\beta}: P \rightarrow M$ that $\delta$-lifts $c \circ \beta$. Since $c: N(K) \rightarrow K$ is $U V^{\ell}$ for every $\ell$, we can lift the homotopy from $K$ to $N(K)$, completing the first part of the argument. 
The rest of the proof of the claim follows the proof of Lemma 6.4. Given a map $\alpha:\left(D^{s+1}, S^{s}\right) \rightarrow(N(K)-\stackrel{\circ}{E}, \partial E)$, construct a nullhomotopy in $(N(K), E)$. Use general position to move the homotopy off of $f^{\prime}(M)$ and then retract the nullhomotopy to $(N(K)-\stackrel{\circ}{E}, \partial E)$. This establishes the claim.

Continuing with the proof of the theorem, we choose a fine handlebody structure on $(N(K)-\stackrel{\circ}{E}, \partial E)$ and trade handles to eliminate all handles of index $\leq k+1$. By Quinn [18, Theorem 6.1], this can be accomplished at the cost of raising the size of the remaining handles to at most $D(k) \cdot \delta$ as measured in $B$, where $D(k)$ is a constant. We now map $M$ to $E$, and $E$ onto $N(K)$ as in the proof of Theorem 6.2. This relaxes the control to $C(m, k, d) \cdot \delta$.

\section{Remarks}

(i) This argument works equally well if the target space is a finite-dimensional ANR $X^{d}$. Embed $X$ into $\mathbb{R}^{2 d+1}$, take a mapping cylinder neighborhood $N(X)$ of $X$, and proceed as above. The argument also extends to infinite-dimensional compact ANRs by crossing with the Hilbert cube and triangulating.

(ii) We can remove the dependency of the constant $C(m, k, d)$ on $m$ and $d$. Take a fine subdivision of $N(K)-\stackrel{\circ}{E}$ and obtain a small handlebody decomposition by taking regular neighborhoods of vertices in the second derived. Given $\mu>0$, we can find a $U V^{k}$ map from $\partial E \times I \cup\{0$-handles $\} \cup \ldots \cup\{(k+2)-$ handles $\}$ to $N(K)$ that is $\mu$-close to the inclusion over $K$. Since handle trading through dimension $k+1$ only reorganizes the handle structure on $\partial E \times I \cup\{0-$ handles $\} \cup$ $\ldots \cup\{(k+2)-$ handles $\}$, we can trade handles and obtain a $U V^{k}$ map from $\partial E \times I$ onto $\partial E \times I \cup\{0$-handles $\} \cup \ldots \cup\{(k+2)-$ handles $\}$ whose control depends on $\delta$ and $k$. The composition is a $U V^{k}$ map whose control only depends on $k$ and $\delta$.

(iii) There is a relative version of Theorem 6.5 which will be important in the sequel. If $f: M \rightarrow K$ is $U V^{k}(\delta)$ and $f \mid \partial M: \partial M \rightarrow K$ is $U V^{k}$, then $f$ is controlled homotopic to a $U V^{k}$-map rel boundary. To see this, attach copies of $\partial M \times[0, \infty)$ and $K \times[0, \infty)$ to $M$ and $K$ and extend levelwise using the restriction of $f$. Let the metrics grow at $t \rightarrow \infty$. Applying the proper version of Theorem 6.5 to this situation, we get a $U V^{k}$ approximation to $f$ that can be extended by $f \mid$ to a $U V^{k}$-map $M \cup \partial M \times[0, \infty] \rightarrow K \times[0, \infty]$ composing with the projection to $K$ gives the desired map.

We close this section with an application of Theorem 6.5. We show that if $M^{n}$ is a compact manifold, $K$ is a polyhedron, and $f: M \rightarrow K$ is a map whose homotopy fiber has finite skeleta (ie, is homotopy equivalent to a $C W$ complex with finite $n$ 
skeleton, for every $n$ ), then $f$ is homotopic to a map with the approximate homotopy lifting property for polyhedra of dimension $\leq\left[\frac{n-3}{2}\right]$.

Definition 6.6 Let $B$ be a metric space. A map $p: E \rightarrow B$ has the approximate homotopy lifting property $(A H L P)$ with respect to a compact space $Z$ if for any homotopy $f: Z \times I \rightarrow B$, map $F_{0}: Z \rightarrow E$ with the property that $p \circ F_{0}=f \mid Z \times$ $\{0\}$, and $\epsilon>0$, there is a map $F: Z \times I \rightarrow E$ such that $F_{0}=F \mid Z \times\{0\}$ and $d(p \circ F(z, t), f(z, t))<\epsilon$, for each $(z, t) \in Z \times I$.

Theorem 6.7 Let $M$ be a compact topological $n$-manifold and $K$ a compact polyhedron. If $f: M \rightarrow K$ is a map whose homotopy fiber has finite skeleta, then $f$ is homotopic to a map with the approximate homotopy lifting property with respect to compact polyhedra of dimension $\leq\left[\frac{n-3}{2}\right]$.

Proof Let $V$ be a mapping cylinder neighborhood of $M$ in some large euclidean space with projection $p: V \rightarrow M$. The homotopy fiber of the composition $f \circ p: V \rightarrow K$ has finite skeleta, since $p$ is cell-like. By Ferry [11, Theorem 2'], there is a polyhedron $V^{\prime}$, a $C E-P L$ map $c: V^{\prime} \rightarrow V$ and a $P L$ map $f^{\prime}: V^{\prime} \rightarrow K$ such that $f^{\prime}$ has the $A H L P$ for compacta of dimension $\leq\left[\frac{n-3}{2}\right]$ and $f \circ p \circ c \simeq f^{\prime}$.

Let $\gamma^{\prime}: M \rightarrow V^{\prime}$ be a homotopy inverse to $p \circ c$. By Theorem 6.5, $\gamma^{\prime}$ is homotopic to a $U V^{k}$ map $\gamma$, where $k=\left[\frac{n-3}{2}\right]$. Notice that since $p \circ c$ is $C E$, we can choose $\gamma$ so that the homotopy $p \circ c \circ \gamma \simeq 1_{M}$ has tracks whose diameters are as small as we wish.

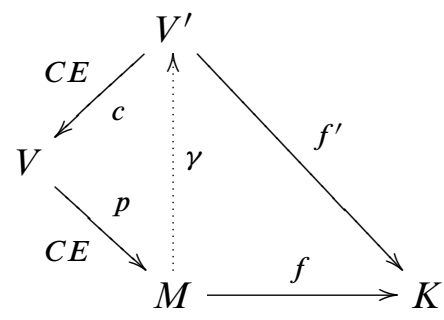

The composition $f^{\prime} \circ \gamma$ is homotopic to $f$, since $f^{\prime} \circ \gamma \simeq(f \circ p \circ c) \circ \gamma=f \circ(p \circ c \circ \gamma) \simeq$ $f \circ 1_{M}=f$. Moreover, $f^{\prime} \circ \gamma$ has the desired approximate homotopy lifting property for polyhedra of dimension $\leq k$, since $\gamma$ is $U V^{k}$ and $f^{\prime}$ has the $A H L P$ for compacta of dimension $\leq k$.

\section{The $U V^{1}$-approximation property}

Definition 7.1 (i) A space $X$ is said to have the $U V^{1}$-approximation property if for every $\epsilon>0$, there is a $\delta>0$ such that every map $f: X \rightarrow K$ to a polyhedron $K$ that is $U V^{1}(\delta)$ over some metric space $B$ is $\epsilon$-homotopic over $B$ to a $U V^{1}$ map. 
(ii) A space $X$ is said to have the linear $U V^{1}$-approximation property if there is a constant $C_{X}>0$ such that every map $f: X \rightarrow K$ to a polyhedron $K$ that is $U V^{1}(\delta)$ over some metric space $B$ is $C_{X} \cdot \delta$-homotopic over $B$ to a $U V^{1}$ map.

In dimensions $\geq 6$, we will characterize excellent metric spaces as ANR homology $n$-manifolds with the $U V^{1}$-approximation property.

Lemma 7.2 Let $X^{n}$ be a compact metric ANR homology $n$-manifold $X, n \geq 6$. Given $\epsilon>0$, there is a pleasant $\epsilon$-controlled 2-patch space $\phi: X_{\epsilon} \rightarrow X$ such that $\phi$ is an $\epsilon$-homotopy equivalence with respect to the control map $1_{X}: X \rightarrow X$.

Proof This is essentially contained in [4]. Start with a degree-one normal map $\psi: M \rightarrow X$, where $M$ is a topological manifold. The existence of $\psi$ is guaranteed by Ferry-Pedersen [12]. There is a $\mu_{0}>0$ such that, for any $0<\mu<\mu_{0}$, the obstruction to doing surgery on $\psi$ to a $\mu$-equivalence is an element $\sigma=\sigma(\psi) \in H_{n}\left(X, \mathbb{L}_{*}(e)\right.$ ) (see Pedersen-Quinn-Ranicki [17] and Ferry [10]). After surgery below middle dimension, using Theorem 6.5, we may assume that $\psi$ is $U V^{1}$.

Take a fine triangulation of $M$ and let $T$ be a very thin regular neighborhood of the 2-skeleton of $M$ in this triangulation. Let $C$ be the closure of the complement of $T$ in $M$.

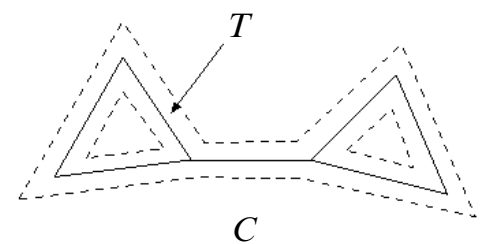

Using the controlled Bestvina-Walsh theorem, after a small homotopy, we may also assume that $\psi|T, \psi| C$ and $\psi \mid \partial T$ are all $U V^{1}$ maps.

Using the element $-\sigma \in H_{*}(X, \mathbb{L}(e))$, do Wall realization on the identity map of $V_{0}=\partial T=\partial C$. This produces a cobordism $\left(V, V_{0}, V_{1}\right)$ and a degree-one normal map $g: V \rightarrow V_{0} \times I$ such that

(i) $g \mid V_{0}=1_{V_{0}}$;

(ii) $f=g \mid V_{1}$ is a fine homotopy equivalence from $V_{1}$ to $V_{0}$ (the control can be as good as we like over $X$ and we may assume that this map is $U V^{1}$ );

(iii) The controlled surgery obstruction of $g$ relative to the boundary is $-\sigma$. 
Form the space $X_{\epsilon}^{\prime}$ by pasting $V$ to $C$ along $V_{0}$ and to $T$ using the controlled equivalence $f=g \mid V_{1}: V_{1} \rightarrow V_{0}=\partial T$. The map $\psi: M \rightarrow X$ "extends" over $V$ to a map $\psi^{\prime}: X_{\epsilon}^{\prime} \rightarrow X$ with trivial surgery obstruction. Do controlled surgery on $\psi^{\prime}$ to obtain a fine homotopy equivalence $\phi: X_{\epsilon} \rightarrow X$. Notice that since all surgeries are performed away from $T, X_{\epsilon}$ is a space of the form $S \cup_{f} T$, where $S$ is a compact manifold. The degree of control for the equivalence $\phi$ depends on two things - the control on the homotopy equivalence $f=g \mid V_{1}$ and the fineness of the initial triangulation of $M$. The regular neighborhood $T$ of the 2-skeleton and the succeeding $U V^{1}$-maps should be constructed so that we can push codimension three polyhedra in $M$ and $X_{\epsilon}^{\prime}$ off of $T$ by homotopies that have small tracks in $X$. The map $\phi: X_{\epsilon} \rightarrow X$ is already $U V^{1}$ when restricted to $T$ and $\partial T$. After a small deformation, we may assume that it is an $\epsilon$-equivalence that restricts to $U V^{1}$ maps on the patches of $X_{\epsilon}$.

Theorem 7.3 Let $X$ be an $n$-dimensional compact metric space, $n \geq 6 . X$ is excellent if and only if $X$ is an ANR homology $n$-manifold with the $U V^{1}$-approximation property.

Proof $(\Rightarrow)$ By Proposition 5.4, $X$ is an ANR homology $n$-manifold. Hence, it suffices to show that $X$ has the $U V^{1}$-approximation property. Let $f: X \rightarrow K$ be a $U V^{1}(\delta)$ map over $B$, for some $\delta>0$. Write $X=\lim \left(X_{i}, \alpha_{i}\right)$ as in the definition of excellent metric spaces, and let $p_{i}: X \rightarrow X_{i}$ denote the projection onto $X_{i}$ and $\gamma_{i}: X_{i} \rightarrow X$ denote the restriction to $X_{i}$ of the retraction $\gamma: T_{X} \rightarrow X$ constructed in Section 3.

For sufficiently large $i, f \circ \gamma_{i}$ is a $U V^{1}(\delta)$ map, so $f^{\prime}=f \circ \gamma_{i} \circ \alpha_{i+1}: X_{i+1} \rightarrow K$ is $U V^{1}(\delta)$ as well. Write $X_{i+1}=S_{i+1} \cup_{f_{i+1}} T_{i+1}$ as in the definition of excellent metric spaces. Since $\alpha_{i+1} \mid \partial T_{i+1}$ is $U V^{1}, f \circ \gamma_{i} \circ \alpha_{i+1} \mid \partial T_{i+1}$ is $U V^{1}(\delta)$ and can be $C(1) \delta$-approximated by a $U V^{1}$ map. By the estimated homotopy extension theorem (see [4]), this homotopy can be extended to a $C(1) \delta$-homotopy of the map $f \circ \gamma_{i} \circ \alpha_{i}$ to a map $f^{\prime}: X_{i+1} \rightarrow K$ that is $U V^{1}((2 C(1)+1) \delta)$ and restricts to a $U V^{1}$ map on $\partial T_{i+1}$. Using the relative version of the controlled Bestvina-Walsh theorem, we can $\left(2 C^{2}(1)+C(1)\right) \delta$-deform $f^{\prime}$ over the patches of $X_{i+1}$ to a $U V^{1}$ map $f^{\prime \prime}: X_{i+1} \rightarrow K$. Notice that $d\left(f \circ \gamma_{i} \circ \alpha_{i+1}, f^{\prime \prime}\right) \leq\left(2 C^{2}(1)+2 C(1)\right) \delta$. Here, $C(1)$ is the constant for $U V^{1}$ approximations as in the remark following the proof of Theorem 6.5.

Let $\bar{f}$ denote the $U V^{1}$ map $\bar{f}=f^{\prime \prime} \circ p_{i+1}$. We claim that, for $i$ large enough, $d(f, \bar{f})<\left(2 C^{2}(1)+2 C(1)+1\right) \delta$. Indeed, first observe that $d\left(f, f \circ \gamma_{i} \circ \alpha_{i+1} \circ p_{i+1}\right)<$ 
$\delta$, for $i$ large enough. Then,

$$
\begin{aligned}
d\left(f, f^{\prime \prime} \circ p_{i+1}\right) & \leq d\left(f, f \circ \gamma_{i} \circ \alpha_{i+1} \circ p_{i+1}\right)+d\left(f \circ \gamma_{i} \circ \alpha_{i+1} \circ p_{i+1}, f^{\prime \prime} \circ p_{i+1}\right) \\
& \leq \delta+d\left(f \circ \gamma_{i} \circ \alpha_{i+1}, f^{\prime \prime}\right) \\
& \leq \delta+\left(2 C^{2}(1)+2 C(1)\right) \delta=\left(2 C^{2}(1)+2 C(1)+1\right) \delta .
\end{aligned}
$$

The map $\bar{f}$ is the desired $U V^{1}$ approximation to $f$.

$(\Leftarrow) \quad$ By Lemma 7.2 and Lemma 2.5, and the assumption that $X$ has the $U V^{1}-$ approximation property, given any $\epsilon>0$, we can construct a pleasant $\epsilon$-controlled 2-patch space $X_{\epsilon}$ and a $U V^{1} \epsilon$-equivalence $f_{\epsilon}: X_{\epsilon} \rightarrow X$ with $U V^{1} \epsilon$-inverse $g_{\epsilon}: X \rightarrow X_{\epsilon}$. We may assume that $f_{\epsilon}$ restricts to $U V^{1}$ maps on the patches of $X_{\epsilon}$. Thus, given a sequence $\left\{\epsilon_{i}\right\}$, we can construct an inverse system

$$
X \longleftarrow \stackrel{f_{1}}{\longleftarrow} X_{1} \stackrel{g_{1}}{\longleftarrow} X \stackrel{f_{2}}{\longleftarrow} X_{2} \stackrel{g_{2}}{\longleftarrow} X \stackrel{f_{3}}{\longleftarrow} X_{3} \stackrel{g_{3}}{\longleftarrow} X \longleftarrow \cdots
$$

such that the $f_{i}$ s and $g_{i} \mathrm{~s}$ are $\epsilon_{i}$-homotopy inverses in the system metric (with properties similar to $f_{\epsilon}$ and $g_{\epsilon}$ ). By Brown [2, Theorem 5.1], the maps $f_{i}$ and $g_{i}$ can be chosen so that the inverse limit of the system is homeomorphic to $X$, since each $f_{i} \circ g_{i}$ is finely homotopic to $1_{X}: X \rightarrow X$ in the system metric. Passing to the subsequence

$$
X_{1} \stackrel{g_{1} \circ f_{2}}{\longleftarrow} X_{2} \stackrel{g_{2} \circ f_{3}}{\longleftarrow} X_{3} \stackrel{g_{3} \circ f_{4}}{\longleftarrow} X_{4} \longleftarrow \cdots
$$

exhibits $X$ as an excellent metric space, provided that $\sum \epsilon_{i}<\infty$.

The following results are consequences of the proof of Theorem 7.3.

Corollary 7.4 If a compact ANR homology $n$-manifold, $n \geq 6$, has the $U V^{1}-$ approximation property, then it has the linear $U V^{1}$-approximation property.

Corollary 7.5 If $X$ is an excellent compact metric space, then for any sequence $\left\{\epsilon_{i}\right\}$ with $\sum \epsilon_{i}<\infty, X$ can be written as the inverse limit of an inverse sequence $\underset{\leftarrow}{\lim }\left(X_{i}, \alpha_{i}\right)$ that exhibits $X$ as an excellent metric space, where each $X_{i}$ is a pleasant $\overleftarrow{\epsilon_{i-1}}$-controlled 2-patch space over $X_{i-1}$.

\section{Some controlled topology}

We begin with a control improvement theorem for maps defined on pleasant patch spaces. This result improves control in two ways, by lifting control over $\mathrm{X}$ to control over $\mathrm{B}$, and by improving the control from size $\delta$ to size $\mu$. 
Theorem 8.1 Suppose that $n \geq 6$ and that we are given a compact ENR $X$. Then for every $\epsilon>0$ there is a $\delta>0$ so that for every $\mu>0$, if we are given a compact ENR $B$ and a $U V^{1}$ homotopy equivalence $q: B \rightarrow X$, there exists $\eta_{1}$, such that if we are given a diagram

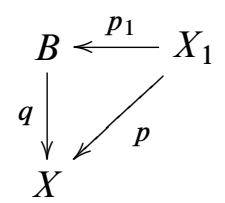

where $p_{1}: X_{1} \rightarrow B$ is an $n$-dimensional $\eta_{1}$-Poincaré duality space over $B$ and $p=q \circ p_{1}$, then there exists $\eta_{2}$ so that if $f: X_{2} \rightarrow X_{1}$ is an $n$-dimensional pleasant $\eta_{2}$-controlled 2-patch space over $X_{1}$ and a $\delta$-homotopy equivalence over $X$, then $f$ is $\epsilon$-homotopic over $X$ to a map $f_{\mu}$ that is a $U V^{1} \mu$-homotopy equivalence over $B$. Moreover, $f_{\mu}: X_{2} \rightarrow X_{1}$ is a pleasant $n$-dimensional $\eta_{2}$-controlled 2-patch space over $X_{1}$

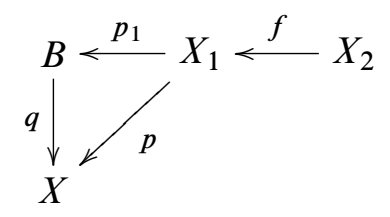

Proof By the stability theorem for controlled surgery parameterized by $U V^{1}$ maps, $[10 ; 17]$, there is a well-defined surgery obstruction $\sigma(f) \in H_{n}(B ; \mathbb{L})$ for sufficiently small $\eta_{1}$ and $\eta_{2}$. The vanishing of $\sigma(f)$ implies that $f$ is normally cobordant to a $\mu$-equivalence over $B$. This is proven in $[10 ; 17]$ for the case in which $X_{2}$ is a manifold and the modification for 2-patch spaces such as $X_{2}$ was discussed in [4]. The basic idea is that surgery involves manipulations up to the middle dimension and in a space such as $X_{2}$ all spheres can be pushed off of the singular set by small moves. At this stage of the construction, the constants $\eta_{i}$ depend only on $B$ and $\mu$.

Since $f: X_{2} \rightarrow X_{1}$ is a $\delta$-homotopy equivalence over $X$, its controlled surgery obstruction is zero in $H_{n}(X ; \mathbb{L})$, provided that $\delta$ is small enough, depending on $B, q$, and $X$. Since $q$ is a $\mathrm{UV}^{1}$ homotopy equivalence, naturality of surgery obstructions implies that $\sigma(f) \in H_{n}(B ; \mathbb{L}) \cong H_{n}(X ; \mathbb{L})$ is also zero. Hence, we can perform surgery on $f$ away from the singular set to obtain a (singular) normal bordism

$$
F:\left(V_{1}, X_{2}, X_{2}^{\prime}\right) \rightarrow\left(X_{1} \times I, X_{1} \times\{0\}, X_{1} \times\{1\}\right),
$$

where $\left.F\right|_{X_{2}}=f$ and where $f_{1}=\left.F\right|_{X_{2}^{\prime}}: X_{2}^{\prime} \rightarrow X_{1}$ is a $\mu$-homotopy equivalence over $B$.

We may assume that $\mu$ is small enough so that both $f$ and $f_{1}$ are $\delta$-homotopy equivalences over $X$. Let $\sigma \in H_{n+1}(X ; \mathbb{L})$ be the controlled surgery obstruction of 
$F$ rel $X_{2} \sqcup X_{2}^{\prime}$. Using the stability of simply-connected controlled surgery groups and the Wall realization procedure [22], we can construct a normal bordism

$$
G:\left(V_{2}, X_{2}^{\prime}, X_{2}^{\prime \prime}\right) \rightarrow\left(X_{1} \times I, X_{1} \times\{1\}, X_{1} \times\{2\}\right),
$$

such that $\left.G\right|_{X_{2}^{\prime}}=f_{1}, f_{2}^{\prime}=\left.G\right|_{X_{2}^{\prime \prime}}: X_{2}^{\prime \prime} \rightarrow X_{1}$ is a $\mu$-equivalence over $B$, and $\sigma(G)=$ $-\sigma$ as an element of $H_{n+1}(B ; \mathbb{L})$. Stacking $V_{1}$ and $V_{2}$ we obtain a normal map

$$
H:\left(V, X_{2}, X_{2}^{\prime \prime}\right) \rightarrow\left(X_{1} \times I, X_{1} \times\{0\}, X_{1} \times\{2\}\right),
$$

where $\left.H\right|_{X_{2}}=f$ and $H_{X_{2}^{\prime \prime}}=f_{2}$ is a $\mu$-equivalence over $B$, and $\sigma(H) \in H_{n+1}(X ; \mathbb{L})$ is zero.

Notice that all constructions were performed away from the singularities of $X_{2}$ so that we may assume that $V$ is a product on a small neighborhood $N$ of the singular set of $X_{2}$. We may also assume that $M=X_{2} \backslash \stackrel{\circ}{N}$ is a compact manifold. Since $0=\sigma(H) \in H_{n+1}(X ; \mathbb{L})$, we can do surgery on $H$ away from $N \times I$ rel $X_{2} \sqcup X_{2}^{\prime \prime}$ and assume that $V$ is a finely controlled $h$-cobordism over $X$ rel $\partial M \times I$. A simple general position argument shows that $V \backslash(N)$ is also a controlled $h$-cobordism over $X$, which, by the thin $h$-cobordism theorem [18], admits an $\epsilon$-product structure rel $\partial M \times I$ if $\delta$ is small enough. Gluing $N \times I$ back in, we obtain an $\epsilon$-deformation over $X$ of the map $f$ to a $\mu$-homotopy equivalence over $B$ as desired.

\section{Resolution of singularities}

Theorem 9.1 If $X$ is a compact ANR homology $n$-manifold, $n \geq 6$, then there exist an excellent ANR homology $n$-manifold $Y$ and a cell-like map $\phi: Y \rightarrow X$.

Proof Let $X$ be a compact ENR homology manifold of dimension $\geq 6$. Our goal is to construct a diagram

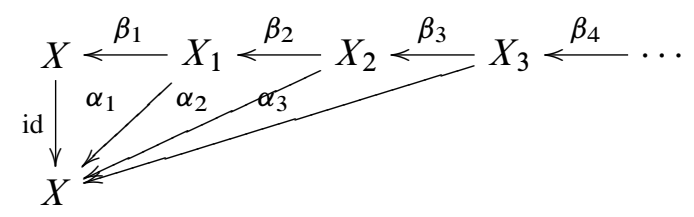

so that for $i \geq 2$ there are sequences $\left\{\epsilon_{i}\right\},\left\{\tau_{i}\right\},\left\{\gamma_{i}\right\}$ of positive numbers so that the following hold.

(i) Each $\beta_{i}$ is $U V^{1} \epsilon_{i-1}$-equivalence over $X_{i-2}$ in the system metric. This guarantees that each $\beta_{i}$ is an $\epsilon_{i-1}+1 / 2^{i-1}$ over $X_{i-1}$.

(ii) Each $X_{i}$ is a pleasant $\epsilon_{i}$-controlled 2-patch space over $X_{i-1}$. 
(iii) $d\left(\alpha_{i-1} \circ \beta_{i}, \alpha_{i}\right)<\tau_{i-1}$.

(iv) Each $\alpha_{i}$ is a $U V^{1} \gamma_{i}$-equivalence.

(v) $\sum \epsilon_{i}<\infty, \sum \tau_{i}<\infty$, and $\lim \gamma_{i}=0$.

This will prove the theorem. The inverse limit $Z=\lim \left\{X_{i}, \beta_{i}\right\}$ is an excellent metric space, so by Proposition 5.4, $Z$ is an ANR homology manifold with the disjoint disk property. For each $i$, there is a projection $p_{i}: Z \rightarrow X_{i}$. By condition (iii), the maps $\alpha_{i} \circ p_{i}$ converge to a map $\alpha: Z \rightarrow X$. By condition (iv), $\alpha$ is an $\epsilon$-equivalence for every $\epsilon>0$, so by Lemma 2.3, $\alpha$ is cell-like. Let $\left\{\epsilon_{i}\right\}$ be any sequence of positive numbers with $\sum \epsilon_{i}<\infty$.

Letting $\epsilon=\epsilon_{1}$ and $\mu=\epsilon_{2}$ in Theorem $8.1^{1}$, there is a $\delta_{1}>0$ so that there exists $\eta_{1}$, such that if we are given a diagram

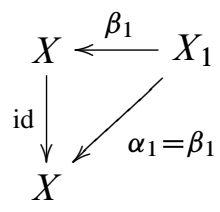

where $\beta_{1}: X_{1} \rightarrow X$ is an $n$-dimensional $\eta_{1}$-Poincaré duality space over $X$, then there exists $\eta_{2}^{\prime}$ so that if $f_{1}: X_{2} \rightarrow X_{1}$ is an $n$-dimensional $\eta_{2}^{\prime}$-controlled 2-patch space over $X_{1}$ and a $\delta_{1}$-equivalence over $X$, then $f_{1}$ is $\epsilon_{1}$-homotopic over $X$ to a $U V^{1} \epsilon_{2}$-homotopy equivalence over $X$.

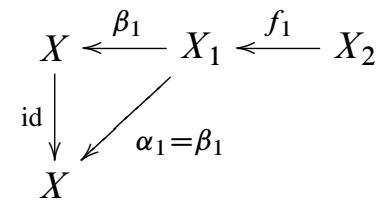

We choose $X_{1}$ and $\beta_{1}: X_{1} \rightarrow X$ so that $\beta_{1}$ is a $U V^{1} \delta_{1}$-equivalence and so that $X_{1}$ is $\eta_{1}$-Poincaré over $X$. We set $\alpha_{1}=\beta_{1}$. Here, we may assume that $\delta_{1}<\epsilon_{0}$. In choosing this and future $\delta_{i}$, we should take care that $\sum \delta_{i}<\infty$.

Letting $\epsilon=\epsilon_{2}$ and $\mu=\epsilon_{3}$ in Theorem 8.1, there is a $\delta_{2}>0$ so that there exists $\eta_{2}^{\prime \prime}$, such that if we are given a diagram

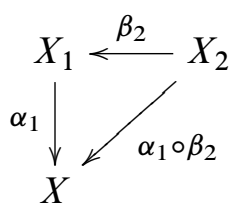

\footnotetext{
${ }^{1}$ This case of Theorem 8.1 is degenerate, but applying the theorem syntactically helps to establish the pattern of our induction, especially in choosing the constants $\eta_{1}$.
} 
where $\beta_{2}: X_{2} \rightarrow X_{1}$ is an $n$-dimensional $\eta_{2}^{\prime \prime}$-Poincaré duality space over $X_{1}$, then there exists $\eta_{3}^{\prime}$ so that if $f_{2}: X_{3} \rightarrow X_{2}$ is an $n$-dimensional $\eta_{3}^{\prime}$-controlled 2-patch space over $X_{2}$ and a $\delta_{2}$-equivalence over $X$, then $f_{2}$ is $\epsilon_{2}$-homotopic over $X$ to a $U V^{1} \epsilon_{3}$-homotopy equivalence over $X_{1}$.

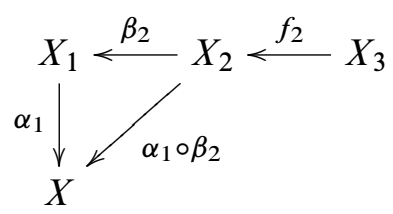

We set $\eta_{2}=\min \left(\eta_{2}^{\prime}, \eta_{2}^{\prime \prime}\right)$. We have now collected enough data to proceed with the construction of $X_{2}, \alpha_{2}$, and $\beta_{2}$. Our construction is a modification of the construction in Lemma 7.2.

Start with a degree-one normal map $\psi: M \rightarrow X$, where $M$ is a topological manifold and do surgery below the middle dimension on $\psi$. There is a $\omega_{0}>0$ such that, for any $0<\omega<\omega_{0}$, the obstruction to doing surgery on $\psi$ to a $\omega$-equivalence is an element $\sigma=\sigma(\psi) \in H_{n}\left(X, \mathbb{L}_{*}(e)\right)$.

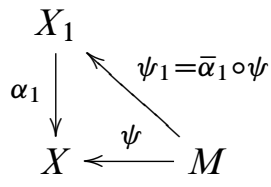

Let $\bar{\alpha}_{1}$ be a $\delta_{1}$-inverse for $\alpha_{1}$ and let $\psi_{1}=\bar{\alpha}_{1} \circ \psi$. The map $\psi_{1}$ is homotopic to a $U V^{1}$-map, so we replace $\psi_{1}$ by a $U V^{1}$-map and replace $\psi$ by $\alpha_{1} \circ \psi_{1}$.

Take a fine triangulation of $M$ and let $T$ be a very thin regular neighborhood of the 2-skeleton of $M$ in this triangulation. Let $C$ be the closure of the complement of $T$ in $M$.

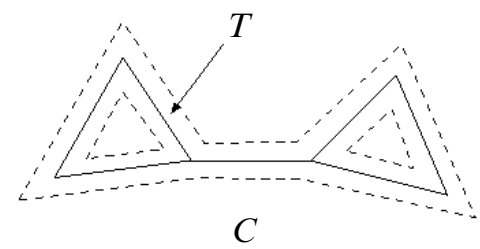

Using the controlled Bestvina-Walsh theorem, after a small homotopy, we may also assume that $\psi|T, \psi| C$ and $\psi \mid \partial T$ are all $U V^{1}$ maps. Since $\alpha_{1}$ is a homotopy equivalence, there is an element $\sigma^{\prime} \in H_{*}\left(X_{1}, \mathbb{L}(e)\right)$ such that $\alpha_{1 *}\left(\sigma^{\prime}\right)=\sigma \in H_{*}(X, \mathbb{L}(e))$.

Using the element $-\sigma^{\prime} \in H_{*}\left(X_{1}, \mathbb{L}(e)\right)$, do Wall realization on the identity map of $V_{0}=\partial T=\partial C$. Proceed as in the proof of Lemma 7.2 to produce a 2-patch space $X_{2}^{\prime}$ with a $U V^{1}$-map $\psi^{\prime}: X_{2}^{\prime} \rightarrow X_{1}$ such that the controlled surgery obstruction of $\alpha_{1} \circ \psi^{\prime}$ 
is zero. We can arrange that $X_{2}^{\prime}$ is a $\eta_{2}$-Poincaré duality space over $X_{1}$. Do surgery on $\alpha_{1} \circ \psi^{\prime}: X_{2}^{\prime} \rightarrow X$ to obtain a 2-patch space $X_{2}$ and an equivalence $\alpha_{2}: X_{2} \rightarrow X$ that is at least a $\delta_{2}$-equivalence and is, in any case, so fine that $\bar{\alpha}_{1} \circ \alpha_{2}: X_{2} \rightarrow X_{1}$ is a $\delta_{1}$-equivalence over $X$. We have a diagram

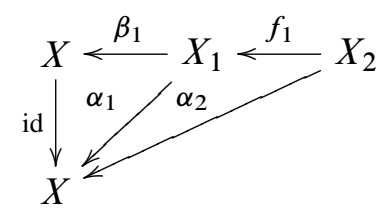

where $f_{1}=\bar{\alpha}_{1} \circ \alpha_{2}$. The surgeries that produced $X_{2}$ from $X_{2}^{\prime}$ were performed on the complement of the set $T$, so by estimated homotopy extension, we can arrange that $f_{1}=\psi^{\prime}$ on a small neighborhood of $T$. Since the Poincare duality of $X_{2}$ over $X_{1}$ depends only on the degree of control of the gluing map on the singular set, $X_{2}$ is an $\eta_{2}$-Poincaré duality space over $X_{1}$.

By our choices of $\delta_{1}$ and $\eta_{2}$, the map $f_{1}$ is $\epsilon_{1}$-homotopic over $X$ to a $U V^{1}$-map $\beta_{2}: X_{2} \rightarrow X_{1}$ that is an $\epsilon_{2}$-equivalence over $X .^{2}$

Letting $\epsilon=\epsilon_{3}$ and $\mu=\epsilon_{4}$ in Theorem 8.1, there is a $\delta_{3}>0$ so that there exists $\eta_{3}^{\prime \prime}$, such that if we are given a diagram

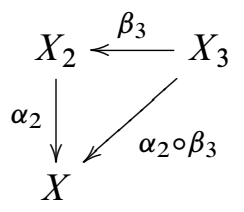

where $\beta_{3}: X_{3} \rightarrow X_{2}$ is an $n$-dimensional $\eta_{3}^{\prime \prime}$-Poincaré duality space over $X_{2}$, then there exists $\eta_{4}^{\prime}$ so that if $f_{3}: X_{4} \rightarrow X_{3}$ is an $n$-dimensional $\eta_{4}^{\prime}$-controlled 2-patch space over $X_{3}$ and a $\delta_{3}$-equivalence over $X$, then $f_{3}$ is $\epsilon_{3}$-homotopic over $X$ to a $U V^{1} \epsilon_{4}$-homotopy equivalence over $X_{2}$.

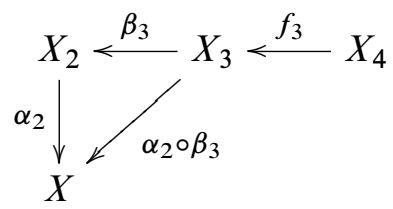

We set $\eta_{3}=\min \left(\eta_{3}^{\prime}, \eta_{3}^{\prime \prime}\right)$. As in the previous stage of the construction, we obtain a 2-patch space $X_{3}$ with an at least $\delta_{3}$ fine $U V^{1}$ homotopy equivalence $\alpha_{3}: X_{3} \rightarrow X$ and a $U V^{1}$ map $f_{2}: X_{3} \rightarrow X_{2}$ so that $f_{2}$ is a $\delta_{2}$-equivalence over $X$ and so that

\footnotetext{
${ }^{2}$ Again, this is a degenerate case. The next stage of the construction represents the general inductive step.
} 
$X_{3}$ is $\eta_{3}$-Poincare over $X_{2}$. It follows that $f_{2}$ is $\epsilon_{2}$-homotopic over $X$ to a $U V^{1}$ map $\beta_{3}: X_{3} \rightarrow X_{2}$ that is an $\epsilon_{3}$-equivalence over $X_{1}$. The composition $\alpha_{2} \circ \beta_{3}$ is $\left(\epsilon_{2}+\delta_{2}\right)$-close to $\alpha_{3}$. The rest of the construction follows this same pattern.

The proof of Theorem B is similar to the proof of Theorem 9.1. If the total surgery obstruction of $Y \rightarrow Z$ is zero, then there is a degree one normal map $f: M \rightarrow Y$ so that the controlled surgery obstruction of $f$ lies in the image of $H_{n}(Y, \mathbb{L})$ in $H_{n}(Z, \mathbb{L})$. This obstruction dies if and only if $f$ is normally bordant to an $\epsilon$-equivalence over $Z$ for sufficiently small $\epsilon$. As in the proof of Theorem 9.1, we can kill this obstruction by replacing $M$ by a controlled 2-patch space $Y_{1}$. Iterating this process as in the proof of Theorem 9.1 gives us a diagram

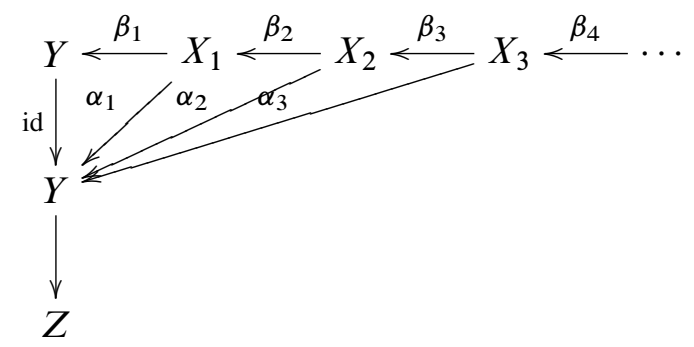

where the maps $\alpha_{i}$ and $\beta_{i}$ satisfy conditions (i)-(v) in the proof of Theorem 9.1. In the limit, we obtain a DDPANR homology manifold $X$ together with a map $g: X \rightarrow Y$ that is an $\epsilon$ equivalence for every $\epsilon>0$. A related result for topological manifolds, was proven by Ferry and Pedersen [12, page 221].

\section{References}

[1] M Bestvina, Characterizing $k$-dimensional universal Menger compacta, Mem. Amer. Math. Soc. 71 (1988) vi+110 MR920964

[2] M Brown, Some applications of an approximation theorem for inverse limits, Proc. Amer. Math. Soc. 11 (1960) 478-483 MR0115157

[3] J L Bryant, General position theorems for generalized manifolds, Proc. Amer. Math. Soc. 98 (1986) 667-670 MR861772

[4] J Bryant, S Ferry, W Mio, S Weinberger, Topology of homology manifolds, Ann. of Math. (2) 143 (1996) 435-467 MR1394965

[5] T A Chapman, Approximation results in topological manifolds, Mem. Amer. Math. Soc. 34 (1981) iii+64 MR634341

[6] T A Chapman, L C Siebenmann, Finding a boundary for a Hilbert cube manifold, Acta Math. 137 (1976) 171-208 MR0425973 
[7] R J Daverman, L S Husch, Decompositions and approximate fibrations, Michigan Math. J. 31 (1984) 197-214 MR752256

[8] J Dugundji, Topology, Allyn and Bacon, Boston (1978) MR0478089

[9] R D Edwards, The topology of manifolds and cell-like maps, from: "Proceedings of the International Congress of Mathematicians (Helsinki, 1978)", Acad. Sci. Fennica, Helsinki (1980) 111-127 MR562601

[10] S Ferry, Epsilon-delta surgery over $\mathbb{Z}$, preprint

[11] S Ferry, A stable converse to the Vietoris-Smale theorem with applications to shape theory, Trans. Amer. Math. Soc. 261 (1980) 369-386 MR580894

[12] S C Ferry, E K Pedersen, Epsilon surgery theory, from: "Novikov conjectures, index theorems and rigidity, Vol. 2 (Oberwolfach, 1993)", London Math. Soc. Lecture Notes 227, Cambridge Univ. Press, Cambridge (1995) 167-226 MR1388311

[13] M W Hirsch, On normal microbundles, Topology 5 (1966) 229-240 MR0198490

[14] C B Hughes, Approximate fibrations on topological manifolds, Michigan Math. J. 32 (1985) 167-183 MR783571

[15] K Kawamura, An inverse system approach to Menger manifolds, Topology Appl. 61 (1995) 281-292 MR1317082

[16] R C Lacher, Cell-like mappings and their generalizations, Bull. Amer. Math. Soc. 83 (1977) 495-552 MR0645403

[17] E K Pedersen, F Quinn, A Ranicki, Controlled surgery with trivial local fundamental groups, from: "High-dimensional manifold topology", World Sci. Publ., River Edge, NJ (2003) 421-426 MR2048731

[18] F Quinn, Ends of maps. I, Ann. of Math. (2) 110 (1979) 275-331 MR549490

[19] F Quinn, Resolutions of homology manifolds, and the topological characterization of manifolds, Invent. Math. 72 (1983) 267-284 MR700771

[20] C P Rourke, B J Sanderson, Introduction to piecewise-linear topology, Springer Study Edition, Springer, Berlin (1982) MR665919

[21] A V Černavskii, A generalization of Keldysh's construction of a monotone map of a cube onto a cube of higher dimension, Russian Math. Surveys 40 (1985) 165-167

[22] C T C Wall, Surgery on compact manifolds, London Math. Soc. Monographs 1, Academic Press, London (1970) MR0431216

JB, WM: Department of Mathematics, Florida State University

Tallahassee FL 32306, USA

SF: Department of Mathematical Sciences, Rutgers University

Piscataway NJ 08854, USA 
SW: Department of Mathematics, The University of Chicago

Chicago IL 60637, USA

bryant@math.fsu.edu, sferry@math.rutgers.edu, mio@math.fsu.edu, shmuel@math. uchicago.edu

http://math.rutgers.edu/ sferry/

Proposed: Rob Kirby

Received: 6 October 2005

Seconded: Peter Teichner and Wolfgang Lueck

Revised: 14 February 2007 